\title{
DESEMPENHO DA TANGERINEIRA 'SPAN AMERICANA' EM DIFERENTES PORTA-ENXERTOS ${ }^{1}$
}

\author{
SIMONE RODRIGUES DA SILVA², EDUARDO SANCHES STUCHI ${ }^{3,4}$, \\ EDUARDO AUGUSTO GIRARDI ${ }^{3}$, TATIANA CANTUARIAS-AVILÉS ${ }^{5}$, \\ MEIRE MENEZES BASSAN ${ }^{6}$
}

RESUMO-Este trabalho objetivou avaliar o crescimento vegetativo, a produção e a qualidade de frutos da tangerina 'Span Americana' em diferentes porta-enxertos, nas condições edafoclimáticas de Bebedouro-SP. O plantio foi realizado em junho de 2003, em espaçamento de $6,0 \mathrm{~m}$ x 3,0 m, sendo utilizada irrigação por gotejamento a partir de 2006. Os porta-enxertos avaliados foram: citranges [Citrus sinensis (L.) Osbeck $\times$ Poncirus trifoliata L. Raf] 'Carrizo' e 'Troyer', tetraploides; trifoliatas (P. trifoliata) 'Davis A' e 'Flying Dragon'; limão Volkameriano Catania 2 (C. volkameriana Tenn. et Pasq.), HRS 849 [(C. aurantium L. cv. 'Smooth Flat Seville' x P. trifoliata cv. 'Argentina')], tangelo 'Orlando' (C. reticulata Blanco $\times$ C. paradisi Macf.) e limão 'Cravo' ( $C$. limonia Osbeck). Foram avaliadas a produção acumulada, a eficiência produtiva e a precocidade de entrada em produção, no período de 2007 a 2009. Avaliaram-se, também, as dimensões das plantas e a taxa média de crescimento das plantas no período de 2005 a 2008, além da qualidade dos frutos em 2006 e 2007. Em pomares irrigados de tangerineira 'Span Americana', os porta-enxertos trifoliata 'Davis A' e HRS 849 apresentam desempenho horticultural satisfatório. Para plantio em alta densidade, a melhor performance da tangerineira 'Span Americana' é obtida com a utilização dos porta-enxertos trifoliata 'Flying Dragon' e citranges 'Troyer' e 'Carrizo'.

Termos para indexação: Citrus reticulata, precocidade, produção, propagação, qualidade.

\section{PERFORMANCE OF 'SPAN AMERICANA' MANDARIN ON DIFFERENT ROOTSTOCKS}

\begin{abstract}
This study aimed to evaluate plant growth and fruit yield and quality of 'Span Americana' mandarin on different rootstocks, under the climatic conditions of Bebedouro, state of São Paulo. The experimental orchard was planted in 2003 using $6 \mathrm{~m}$ x $3 \mathrm{~m}$ spacing, and was drip irrigated since 2006. The following rootstocks were studied: the 'Carrizo' and 'Troyer' tetraploid citranges [Citrus sinensis (L.) Osbeck $\times$ Poncirus trifoliata]; the trifoliates (P. trifoliata) 'Davis A' and 'Flying Dragon: Catania 2 Volkamer lemon (C. volkameriana Tenn. et Pasq.); HRS 849 [(C. aurantium L. cv. 'Smooth Flat Seville' x P. trifoliata cv. 'Argentina')]; 'Orlando' tangelo (C. reticulata Blanco $\times$ C. paradisi Macf.), and 'Cravo' 'Rangpur' lime (C. limonia Osbeck). Mean cumulative yield, productive efficiency and early-bearing yield were calculated for the 2007-2009 period. Plant size and mean plant growth rate was calculated from the annual variation in canopy volume during 2005 through 2008. Fruit quality was assessed in 2006 and 2007. Under irrigated conditions, the 'Davis A' trifoliate and the HRS 849 induced good horticultural performance to 'Span Americana' mandarin and may be used as alternative rootstocks. For high-density plantings, the rootstocks 'Flying Dragon' trifoliate and the 'Troyer' and 'Carrizo' tetraploid citranges may induce better performance to the trees.
\end{abstract}

Index terms: Citrus reticulata, earliness, yield, propagation, quality.

\footnotetext{
${ }^{1}$ (Trabalho 190-13). Recebido em: 15-05-2013. Aceito para publicação em: 08-10-2013.

${ }^{2}$ Universidade de São Paulo, Escola Superior de Agricultura "Luiz de Queiroz”, Caixa Postal 9, CEP 13418-900, Piracicaba, SP. Autor correspondente. E-mail: srsilva@usp.br;

${ }^{3}$ Pesquisador Embrapa Mandioca e Fruticultura-BA. E-mails: stuchi@cnpmf.embrapa.br; girardi@cnpmf.embrapa.br

${ }^{4}$ Pesquisador Estação Experimental de Citricultura de Bebedouro, Bebedouro-SP.

${ }^{5}$ Doutora em Fitotecnia USP/ESALQ. E-mail: tatiana.cantuarias@gmail.com

${ }^{6}$ Mestre em Fitotecnia USP/ESALQ. E-mail meirebassan@yahoo.com.br
} 


\section{INTRODUÇÃO}

A China destaca-se como principal produtor mundial de tangerinas, sendo responsável por $65 \%$ da produção, e o Brasil ocupa a terceira posição, com 1,12 milhão de tonelada da fruta, produzidas principalmente em São Paulo, Paraná, Rio Grande do Sul e Minas Gerais (AGRIANUAL, 2013). Apesar da grande variedade de cultivares e híbridos de tangerineiras existentes (SILVA et al., 2009), predomina em São Paulo o cultivo da tangerineira 'Ponkan' (Citrus reticulata Blanco) em 56,2\% da área, seguido pelo cultivo do tangoreiro 'Murcott' [C. sinensis (L.) Osbeck x C. reticulata] (23\%), da tangerineira 'Cravo' (C. reticulata) $(11,9 \%)$ e da mexeriqueira 'Montenegrina' ( $C$. deliciosa Tennore) (8,6\%) (CASER; AMARO, 2006). Segundo esses autores, nos últimos anos, registrou-se um aumento no percentual de plantio do tangor 'Murcott', em resposta ao aumento na demanda desta variedade pelo mercado paulista.

A 'Span Americana', introduzida da França, é uma variedade com tamanho e coloração adequados ao gosto do consumidor brasileiro, apesar de apresentar 11 sementes por fruto, em média. O sabor e o formato dos frutos são bastante semelhantes aos da tangerina 'Ponkan' tradicional, podendo ser colhidos a partir de março. Assim, podem ser colocados mais precocemente no mercado de fruta fresca (PIO et al., 2001), com um mês de antecedência à safra de tangerinas no sudeste do País, que se concentra entre maio e julho.

As tangerineiras no Brasil encontram-se enxertadas principalmente sobre limoeiro 'Cravo' (C. limonia Osbeck). Esse porta-enxerto destacase por sua capacidade de induzir tolerância à seca às copas nele enxertadas, tolerância ao vírus da tristeza dos citros (CTV), elevado vigor às mudas no viveiro, rápida entrada em produção, compatibilidade com todas as variedades cítricas, alta produção e maturação precoce dos frutos. No entanto, induz frutos de qualidade inferior e maior risco fitossanitário, o que limita seu uso como portaenxerto para produção de frutas de mesa, como as tangerinas (POMPEU JÚNIOR, 2005).

Diante desse cenário, é importante diversificar as combinações entre copas de tangerineiras e diferentes porta-enxertos, visando a obter elevada produção e maior qualidade da fruta, além da possibilidade de extensão do período de safra pela antecipação do início de colheita. Este trabalho objetivou avaliar o crescimento vegetativo, a produção e a qualidade de frutos da tangerineira 'Span Americana' em diferentes porta-enxertos, nas condições edafoclimáticas de Bebedouro-SP.

\section{MATERIAL E MÉTODOS}

O trabalho foi realizado na Estação Experimental de Citricultura de Bebedouro (EECB), em Bebedouro-SP $\left(20^{\circ} 53^{\prime} 16^{\prime \prime} \mathrm{S}, 4^{\circ} 28^{\prime} 11^{\prime \prime} \mathrm{W}\right.$, altitude $601 \mathrm{~m}$ ), em Argissolo Vermelho-Amarelo distrófico típico, de textura média-argilosa e horizonte A moderado (PVA). O clima é do tipo Cwa (subtropical com inverno moderado e seco, verão quente e chuvoso), com precipitação pluviométrica anual de $1.522 \mathrm{~mm}$. Durante o período experimental, a média das temperaturas mínima e máxima foi $17,21^{\circ} \mathrm{C}$ e $29,24^{\circ} \mathrm{C}$, respectivamente, e a precipitação pluviométrica média anual foi de 1.445 $\mathrm{mm}$. O plantio foi realizado em junho de 2003, em espaçamento de $6,0 \mathrm{~m} \times 3,0 \mathrm{~m}$ (555 plantas $\left.\mathrm{ha}^{-1}\right)$. Nos três primeiros anos, o pomar foi conduzido sob sequeiro, passando a ser irrigado por gotejamento, em 2006, sendo manejado de acordo com as práticas culturais recomendadas para a cultura dos citros (MATTOS JÚNIOR et al., 2005).

A tangerineira 'Span Americana' (Citrus reticulata $\mathrm{Blanco}$ ) foi selecionada entre as variedades existentes no BAG-Citros da EECB após resultados preliminares sobre a qualidade dos frutos. Essa variedade foi enxertada nos porta-enxertos citranges 'Carrizo' e 'Troyer' [C. sinensis x Poncirus trifoliata (L.) Raf.] tetraploides, trifoliata 'Davis $\mathrm{A}^{\prime}(P$. trifoliata), limoeiro 'Volkameriano' ( $C$. volkameriana Ten. \& Pasq.) clone Catania 2, HRS 849 [(C. aurantium L. cv. 'Smooth Flat Seville' $\mathrm{x}$ $P$. trifoliata cv. 'Argentina')], tangelo 'Orlando' $(C$. tangerina Tanaka $\mathrm{x} C$. paradisi Macfad.), trifoliata 'Flying Dragon' (P. trifoliata var. monstrosa) e limoeiro 'Cravo' (C. limonia Osbeck).

Entre os meses de abril e maio, obteve-se a produção total de cada planta, pela pesagem dos frutos após a colheita, utilizada para o cálculo da produção acumulada no período de 2007 a 2009. As dimensões das plantas foram avaliadas no período de 2005 a 2008. Calculou-se a taxa média de crescimento (TC) pela variação anual do volume de copa (V), calculado por: $\mathrm{V}=(\pi / 6) \times \mathrm{H} \times \mathrm{D}_{1} \times \mathrm{D}_{\mathrm{r}}$, em que: $\mathrm{H}=$ altura da planta $(\mathrm{m})$ e $\mathrm{D}_{1}$ e $\mathrm{D}_{\mathrm{r}}=$ diâmetros da planta nos sentidos paralelo e perpendicular à linha de plantio (m), respectivamente (ZEKRI, 2000).

A eficiência da produção (EP) foi estimada pela relação entre a produção de frutos por planta e o volume da copa de cada combinação copa e portaenxerto, no ano de 2007. O índice de alternância da produção (IAP) foi calculado entre os anos de 2007 e 2009, usando-se a seguinte fórmula: IAP $=1 / n-1 \times$ 
$\left\{\left|\left(a_{2}-a_{1}\right)\right| /\left(a_{2}+a_{1}\right)+\left|\left(a_{3}-a_{2}\right)\right| /\left(a_{3}+a_{2}\right)+\ldots+\left|\left(a_{(n)}-a_{(n-1)}\right)\right| /\right.$ $\left.\left(\mathrm{a}_{(\mathrm{n})}+\mathrm{a}_{(\mathrm{n}-1)}\right)\right\}$, em que: $\mathrm{n}=$ número de anos e $\mathrm{a}_{1}, \mathrm{a}_{2}$, ..., $\mathrm{a}_{(\mathrm{n}-1)}, \mathrm{a}_{(\mathrm{n})}=$ produção dos anos correspondentes (SMITH et al., 2004). A precocidade de produção (PP) foi calculada percentualmente pela razão entre a produção verificada na primeira safra (2004) e a produção total acumulada nas 3 primeiras safras (2004 a 2007), sendo um índice que permite estimar a antecipação da produção de uma determinada variedade copa enxertada em um determinado portaenxerto.

A análise de qualidade dos frutos foi realizada nos meses de abril/maio em 2006 e 2007, utilizandose da amostra de 10 frutos por planta. A massa dos frutos foi determinada em uma balança com sensibilidade de 5,0 g, e a extração do suco, através de uma extratora OIC, modelo OTTO 1800. O rendimento em suco foi calculado pela relação massa do suco/massa do fruto, dado em percentagem. O teor de sólidos solúveis totais (SST) foi determinado por leitura direta em refratômetro digital (Atago), com os valores corrigidos para $20^{\circ} \mathrm{C}$ e expressos em ${ }^{\circ}$ Brix, e a acidez titulável (AT) foi obtida por titulação de $25 \mathrm{~mL}$ de suco com hidróxido de sódio 0,3125 N. Também foram calculados o ratio, pela relação aritmética entre SST e AT, e o índice tecnológico (IT), obtido pela equação: $\mathrm{IT}=$ (rendimento em suco $\mathrm{x}$ sólidos solúveis x 40,8)/10.000 (CAVALCANTE et al., 2009).

O delineamento foi em blocos ao acaso, com oito repetições e uma planta por parcela. Os dados obtidos foram submetidos à análise de variância, e as médias, agrupadas pelo teste de Scott-Knott $(\mathrm{P}<0,05)$. Para as variáveis produção acumulada $\sqrt{ }\left(\mathrm{kg} \mathrm{planta}^{-1}\right.$ e $\left.\mathrm{t} \mathrm{ha} \mathrm{h}^{-1}\right)$, precocidade de produção, eficiência produtiva média, diâmetro e volume de copa, e taxa média de crescimento, foi realizada a transformação dos dados $(\sqrt{\mathrm{x}+0,5})$ para satisfazer os supostos da análise de variância.

\section{RESULTADOS E DISCUSSÃO}

No período de 2007 a 2009, os portaenxertos limoeiro 'Cravo', trifoliata 'Davis A' e HRS 849 induziram produção acumulada que superou em 43 a $116 \%$ as produções observadas sobre citrangeiros tetraploides 'Carrizo' e 'Troyer', limoeiro 'Volkameriano', tangeleiro 'Orlando' e trifoliata 'Flying Dragon' (Tabela 1). O bom desempenho do limoeiro 'Cravo' quanto à produção de tangerinas já foi observado para diversas outras variedades, como 'Fallglo' [(C. reticulata Blanco $\mathrm{x}(C$. paradisi Macf. x C. reticulata Blanco)] x (C. reticulata $\mathrm{x} C$. sinensis L. Osbeck) (MOURÃO et al., 2007), 'Ponkan' (C. reticulata Blanco) (STENZEL et al., 2003), satsuma 'Okitsu' (C. unshiu Marcow.) (CANTUARIAS-AVILÉS et al., 2010) e 'Fremont' (C. clementina Hort. ex Tan. x C. reticulata Blanco) (NÚNEZ et al., 2007), confirmando seu alto vigor e seu rendimento produtivo (POMPEU JUNIOR, 2005).

Já os trifoliatas têm apresentado desempenhos variados para tangerinas. As seleções de trifoliata 'Flying Dragon', 'EEL'e 'Kryder 8-5' não induziram boa produção às copas de tangor 'Murcott' (FIGUEIREDO et al., 2006), 'Ponkan' (STENZEL et al., 2003) e satsuma 'Okitsu' (CANTUARIASAVILÉS et al., 2010), assim como observado com o trifoliata 'Flying Dragon' neste trabalho (Tabela 1). Contudo, o trifoliata 'Davis A' conferiu alta produção acumulada à tangerineira 'Span Americana', enquanto os citranges 'Carrizo' e 'Troyer' induziram níveis intermediários de produção acumulada até o $6^{\circ}$ ano após o plantio. Na Flórida, o citrange 'Carrizo' também induziu produtividade intermediária à tangerineira 'Fallglo' (BOWMAN, 1998).

$\mathrm{Na}$ avaliação da produção acumulada da tangerineira 'Span Americana', no período de 2007 a 2009 (quarto a sexto ano após o plantio), observouse que os porta-enxertos limoeiro 'Volkameriano' e tangeleiro 'Orlando' induziram 85,33 e 88,60\% da produção total acumulada, respectivamente, enquanto os demais resultaram em menos de $47 \%$ da produção (Tabela 1). Segundo Pompeu Júnior (2001), citros enxertados em limoeiros 'Cravo', 'Rugoso' e 'Volkameriano' tendem a ser mais precoces quanto à entrada em produção. No Paraná, a tangerineira 'Ponkan' foi avaliada sobre sete porta-enxertos, entretanto a precocidade da produção sobre limoeiro 'Volkameriano' foi observada apenas na primeira safra (1992) quando comparado à tangerineira 'Cleópatra' e ao P. trifoliata. Nos anos seguintes de avaliação (1993-99), a produção acumulada foi maior sobre 'Cleópatra' (STENZEL et al., 2003).

Todos os porta-enxertos induziram elevada alternância de produção às plantas de tangerineira 'Span Americana', com valores médios de IAP próximos ou iguais a 1,00 (Tabela 1). A alta alternância produtiva é característica dos citros, e especialmente intensa nas tangerineiras. Em tangerineira 'Ponkan' enxertada sobre sete porta-enxertos, foram relatados menores valores médios de IAP, de 0,28 a 0,46 , entre o $4^{\circ}$ e o $11^{\circ}$ ano após o plantio (STENZEL et al., 2003). Em estudo conduzido durante 26 anos na região subtropical da Austrália, não foi constatado efeito de sete porta-enxertos sobre a alternância de produção do tangor 'Ellendale' (SMITH et al., 2004). Resultados semelhantes também foram observados por Georgiou (2000) ao avaliar a tangerineira 
'Nova' sobre onze porta-enxertos, não observando diferenças no IAP. Por outro lado, CantuariasAvilés et al. (2010), estudando 12 porta-enxertos para tangerineira satsuma 'Okitsu' em BebedouroSP, concluíram que a alternância de produção foi maior sobre trifoliata 'FCAV' e tangelo 'Orlando'. Embora existam evidências em favor da influência do porta-enxerto sobre a bianualidade produtiva de citros (MONSELISE; GOLDSCHMIDT, 1982), os resultados obtidos neste estudo reforçam a ideia de que esse efeito seja menos pronunciado do que aquele exercido pela cultivar copa, especialmente em se tratando de tangerineiras, que apresentam maior fixação de frutos dentre os citros.

Em 2007, os porta-enxertos foram agrupados em duas categorias, de acordo com a eficiência produtiva (EP): i) trifoliata 'Flying Dragon' e citrangeiros tetraploides 'Troyer' e 'Carrizo', de alta $\mathrm{EP}\left(8,88\right.$ a $12,1 \mathrm{~kg}$ fruto $\left.\mathrm{m}^{-3}\right)$, e ii) HRS 849 , tangeleiro 'Orlando', trifoliata 'Davis A' e limoeiros 'Volkameriano' e 'Cravo', de menor EP (6,01 a $8,14 \mathrm{~kg}$ fruto $\mathrm{m}^{-3}$ ) (Tabela 1$)$. Esses valores estão inversamente relacionados com os obtidos para o crescimento da copa, uma vez que o trifoliata 'Flying Dragon' e os citrangeiros, com alta e média EP, respectivamente, induziram as menores dimensões de copa (Tabela 2). A alta EP conferida pelo trifoliata 'Flying Dragon' à tangerineira 'Span Americana' coincide com os efeitos induzidos por esse portaenxerto em tangerineira satsuma 'Okitsu' e 'Oneco' (CANTUARIAS-AVILÉS et al., 2010; GONZATTO, et al., 2011). Por outro lado, os menores valores de EP foram observados em plantas sobre porta-enxertos que conferiram maior tamanho à copa, como HRS 849 e tangeleiro ‘Orlando' (Tabela 1). O porta-enxerto tangeleiro 'Orlando' também induziu baixa EP à copa de tangerineira satsuma 'Okitsu' (CANTUARIASAVILÉS et al., 2010). As maiores alturas das copas de 'Span Americana' foram conferidas pelos limoeiros 'Cravo' e 'Volkameriano', seguidos do HRS 849 e tangeleiro 'Orlando'. Sobre o trifoliata 'Davis A' e citranges tetraploides 'Troyer' e 'Carrizo', as plantas atingiram menores alturas, sendo que as copas mais baixas foram aquelas enxertadas sobre trifoliata 'Flying Dragon' (Tabela 2). Tangerineiras satsuma 'Okitsu' (CANTUARIAS-AVILÉS, 2010) e 'Unshiu' (ABEDI GHESHLAGHI; FIFAEI, 2012) enxertadas sobre trifoliata 'Flying Dragon' apresentaram bom desempenho em plantios em alta densidade. O menor porte e alta EP conferidos às copas da tangerineira 'Span Americana' pelos citrangeiros 'Troyer' e 'Carrizo' e pelo trifoliata 'Flying Dragon' poderiam sugerir a utilização destes porta-enxertos para situações de plantio adensado.
No período de 2005 a 2008, plantas de tangerineira 'Span Americana' enxertadas em tangeleiro 'Orlando' e HRS 489 apresentaram maiores taxas médias de crescimento do que quando enxertadas em trifoliata 'Davis A', e limoeiros 'Cravo' e 'Volkameriano' (Tabela 2). Baixas taxas de crescimento das copas de 'Span Americana' foram observadas sobre os citrangeiros, mas o menor valor foi verificado sobre trifoliata 'Flying Dragon'. O elevado vigor conferido às copas enxertadas sobre limoeiro 'Cravo' e tangeleiro 'Orlando' também foi observado para tangerineiras 'Fairchild' (NÚNEZ et al., 2008), 'Fremont' (NÚNEZ et al., 2007), 'Sunburst' (MOURÃO et al., 2007) e satsuma 'Okitsu' (CANTUARIAS-AVILÉS et al., 2010).

Em relação à qualidade de frutos, verificouse que os porta-enxertos não alteraram a massa dos frutos e o rendimento em suco da tangerina 'Span Americana' (Tabela 3). Efeito similar foi relatado para as tangerineiras 'Sunburst' e 'Fallglo' enxertadas sobre quatro porta-enxertos, em Bebedouro-SP (MOURÃO et al., 2007), e também para tangor 'Murcott' sobre 16 porta-enxertos, em Itirapina-SP (FIGUEIREDO et al., 2006). Já os atributos químicos da qualidade dos frutos de tangerina 'Span Americana' foram influenciados pelos porta-enxertos. Em relação ao teor de SST, os melhores resultados foram obtidos com os portaenxertos citranges tetraploides, trifoliatas 'Davis A' e 'Flying Dragon', limoeiro 'Cravo' e HRS 849 (Tabela 3). Em tangerinas, o elevado teor de SST dos frutos está associado à melhor qualidade do produto, como tem sido constatado em outros trabalhos (CANTUARIAS-AVILÉS et al., 2010; TAKAHARA et al., 2001). Entretanto, os altos teores de SST induzidos pelo limoeiro 'Cravo' aos frutos da tangerineira 'Span Americana' foram significativamente superiores aos obtidos com os porta-enxertos tangeleiro 'Orlando' e limoeiro 'Volkameriano'. Esses resultados contrastam com os baixos teores induzidos pelo limoeiro 'Cravo' aos frutos das tangerineiras satsuma 'Okitsu' (CANTUARIAS-AVILÉS et al., 2010), 'Fallglo' e 'Sunburst' (MOURÃO et al., 2007). No trabalho de Pompeu Júnior et al. (2002), os citrangeiros 'Troyer' e 'Carrizo', e o trifoliata 'Davis A' induziram altos teores de SST aos frutos da laranjeira 'Valência', efeito similar ao observado sobre os frutos da tangerineira 'Span Americana' avaliados neste trabalho (Tabela 3).

Em relação à acidez titulável, os menores valores foram observados nos frutos colhidos sobre os limoeiros 'Cravo' e 'Volkameriano', e, consequentemente, o ratio do suco dos frutos foi 
superior (Tabela 3). Por outro lado, o tangeleiro 'Orlando' e o HRS 849 induziram os menores valores de ratio aos frutos. Apesar de alguns estudos indicarem que o porta-enxerto não influencia na acidez e no ratio em tangerinas (FIGUEIREDO et al., 2006; NÚNEZ et al., 2008), o presente trabalho confirma a indução de precocidade de maturação dos frutos obtida em frutos de tangerinas enxertadas sobre limoeiros 'Cravo' e 'Volkameriano', conforme foi observado para tangerineiras 'Ellendale' e 'Fortune' sobre limoeiro 'Cravo' (STUCHI et al., 2008). Quanto ao índice tecnológico, não se observaram diferenças entre os porta-enxertos no período da avaliação (Tabela 3 ).

TABELA 1- Produção acumulada de frutos, índice de alternância de produção (IAP), precocidade de produção (PP) e eficiência produtiva média (EP) da tangerineira 'Span Americana' enxertada em diferentes porta-enxertos (555 plantas ha' ${ }^{-1}$ ). Bebedouro, SP, 2007-2009.

\begin{tabular}{lcccccccccc}
\hline Porta-enxerto & $\begin{array}{c}\text { Produção } \\
\mathrm{kg} \mathrm{planta}^{-1}\end{array}$ & $\begin{array}{c}\text { Produção } \\
\mathrm{tha}^{-1}\end{array}$ & IAP & \multicolumn{2}{c}{$\begin{array}{c}\text { PP* } \\
\%\end{array}$} & \multicolumn{2}{c}{$\begin{array}{c}\text { EP** } \\
\mathrm{kg} \mathrm{m}^{-3}\end{array}$} \\
\hline Citrange 'Carrizo' tetraploide & 80,91 & $\mathrm{~b}$ & 44,95 & $\mathrm{~b}$ & 0,97 & $\mathrm{a}$ & 44,64 & $\mathrm{~b}$ & 8,88 & $\mathrm{a}$ \\
Citrange 'Troyer' tetraploide & 101,09 & $\mathrm{a}$ & 56,16 & $\mathrm{a}$ & 0,99 & $\mathrm{a}$ & 38,65 & $\mathrm{~b}$ & 9,59 & $\mathrm{a}$ \\
Trifoliata 'Davis A' & 137,59 & $\mathrm{a}$ & 76,44 & $\mathrm{a}$ & 0,98 & $\mathrm{a}$ & 43,57 & $\mathrm{~b}$ & 7,46 & $\mathrm{~b}$ \\
Limoeiro 'Volkameriano' & 63,75 & $\mathrm{~b}$ & 35,42 & $\mathrm{~b}$ & 1,00 & $\mathrm{a}$ & 85,33 & $\mathrm{a}$ & 7,67 & $\mathrm{~b}$ \\
HRS 849 & 144,01 & $\mathrm{a}$ & 80,01 & $\mathrm{a}$ & 0,91 & $\mathrm{a}$ & 38,15 & $\mathrm{~b}$ & 6,01 & $\mathrm{~b}$ \\
Tangelo 'Orlando' & 67,46 & $\mathrm{~b}$ & 37,48 & $\mathrm{~b}$ & 1,00 & $\mathrm{a}$ & 88,60 & $\mathrm{a}$ & 6,94 & $\mathrm{~b}$ \\
Trifoliata 'Flying Dragon' & 70,84 & $\mathrm{~b}$ & 39,36 & $\mathrm{~b}$ & 0,99 & $\mathrm{a}$ & 40,65 & $\mathrm{~b}$ & 12,14 & $\mathrm{a}$ \\
Limoeiro 'Cravo' & 142,16 & $\mathrm{a}$ & 78,98 & $\mathrm{a}$ & 0,99 & $\mathrm{a}$ & 46,62 & $\mathrm{~b}$ & 8,14 & $\mathrm{~b}$ \\
CV (\%) & 23,81 & & 23,66 & 0,02 & 18,76 & & 17,11 \\
\hline
\end{tabular}

Médias seguidas da mesma letra minúscula na coluna pertencem ao mesmo agrupamento pelo teste de Scott-Knott (5\%).

* Porcentagem de produção na primeira safra (2004) em relação ao total acumulado entre 2007 a 2009.

** Safra 2007

TABELA 2- Diâmetro médio, altura e volume de copa de tangerineira 'Span Americana' em diferentes porta-enxertos em 2008, e taxa média de crescimento (TC) de copa no período de 2005 a 2008. Bebedouro, SP.

\begin{tabular}{|c|c|c|c|c|c|c|c|c|}
\hline \multirow{2}{*}{ Porta-enxerto } & \multicolumn{2}{|c|}{ Diâmetro médio* } & \multirow{2}{*}{\multicolumn{2}{|c|}{ Altura }} & \multicolumn{2}{|c|}{ Volume } & \multicolumn{2}{|c|}{$\mathrm{TC}$} \\
\hline & \multicolumn{2}{|c|}{--------- $\mathrm{m}$---------- } & & & \multicolumn{2}{|c|}{$\mathrm{m}^{3}$} & \multicolumn{2}{|c|}{$\mathrm{m}^{3} \mathrm{ano}^{-1}$} \\
\hline Citrange 'Carrizo' tetraploide & 1,74 & $\mathrm{~d}$ & 3,09 & $\mathrm{c}$ & 5,34 & $\mathrm{~b}$ & 1,44 & $\mathrm{c}$ \\
\hline Citrange 'Troyer' tetraploide & 1,83 & $\mathrm{c}$ & 3,08 & $\mathrm{c}$ & 5,45 & $\mathrm{~b}$ & 1,54 & $\mathrm{c}$ \\
\hline Trifoliata 'Davis A' & 2,38 & $\mathrm{c}$ & 3,20 & $\mathrm{c}$ & 9,57 & $\mathrm{a}$ & 3,09 & $\mathrm{~b}$ \\
\hline Limoeiro 'Volkameriano' & 2,23 & $\mathrm{~b}$ & 3,53 & $\mathrm{a}$ & 9,23 & $\mathrm{a}$ & 2,30 & $\mathrm{~b}$ \\
\hline HRS 849 & 2,54 & a & 3,43 & $\mathrm{~b}$ & 11,62 & $\mathrm{a}$ & 3,34 & $\mathrm{a}$ \\
\hline Tangelo 'Orlando' & 2,20 & $\mathrm{~b}$ & 3,31 & $\mathrm{~b}$ & 10,78 & $\mathrm{a}$ & 3,50 & $\mathrm{a}$ \\
\hline Trifoliata 'Flying Dragon' & 1,60 & $\mathrm{~d}$ & 2,73 & $\mathrm{~d}$ & 3,71 & $\mathrm{~b}$ & 0,83 & $\mathrm{~d}$ \\
\hline Limoeiro 'Cravo' & 2,29 & $\mathrm{~b}$ & 3,61 & $\mathrm{a}$ & 9,99 & $\mathrm{a}$ & 2,35 & $\mathrm{~b}$ \\
\hline $\mathrm{CV}(\%)$ & 5,21 & & 3,80 & & 14,48 & & 12,28 & \\
\hline
\end{tabular}

Médias seguidas da mesma letra minúscula na coluna pertencem ao mesmo agrupamento pelo teste de Scott-Knott (5\%).

* Média do diâmetro da copa no sentido paralelo e perpendicular à linha de plantio. 
TABELA 3- Massa do fruto, rendimento em suco (RS), sólidos solúveis totais (SST), acidez titulável (AT), ratio e índice tecnológico (IT) de tangerina 'Span Americana' enxertada em diferentes portaenxertos (2006 e 2007*). Bebedouro, SP.

\begin{tabular}{|c|c|c|c|c|c|c|c|}
\hline Porta-enxerto & $\begin{array}{c}\text { Massa } \\
\mathrm{g}\end{array}$ & $\begin{array}{c}\mathrm{RS} \\
\%\end{array}$ & $\begin{array}{l}\text { SST } \\
{ }^{\circ} \text { Brix }\end{array}$ & & $\begin{array}{l}\text { AT } \\
\%\end{array}$ & Ratio & $\begin{array}{c}\text { IT } \\
\operatorname{kg~SS} * *\end{array}$ \\
\hline Citrange 'Carrizo' tetraploide & $206,17 \mathrm{a}$ & 33,61 a & 9,45 c & $\mathrm{a}$ & $0,60 \mathrm{a}$ & $16,19 \mathrm{~b}$ & $1,30 \mathrm{a}$ \\
\hline Citrange 'Troyer' tetraploide & $223,05 \mathrm{a}$ & 34,74 a & 9,64 & $\mathrm{a}$ & 0,59 a & $16,86 \mathrm{~b}$ & $1,36 \mathrm{a}$ \\
\hline Trifoliata 'Davis A' & $222,30 \mathrm{a}$ & 35,29 a & 9,71 & $\mathrm{a}$ & $0,57 \mathrm{a}$ & $17,18 \mathrm{~b}$ & $1,40 \mathrm{a}$ \\
\hline Limoeiro 'Volkameriano' & $215,40 \mathrm{a}$ & 29,70 a & 8,63 & $\mathrm{c}$ & $0,46 \mathrm{~b}$ & 18,97 a & $1,05 \mathrm{a}$ \\
\hline HRS 849 & $204,88 \mathrm{a}$ & 34,70 a & 9,46 & $\mathrm{a}$ & 0,62 a & $15,35 \mathrm{c}$ & $1,35 \mathrm{a}$ \\
\hline Tangelo 'Orlando' & $190,70 \mathrm{a}$ & 37,42 a & 9,18 & $\mathrm{~b}$ & 0,62 a & $14,98 \mathrm{c}$ & $1,40 \mathrm{a}$ \\
\hline Trifoliata 'Flying Dragon' & $212,35 \mathrm{a}$ & $35,20 \mathrm{a}$ & 10,03 & $\mathrm{a}$ & 0,59 a & $17,34 \mathrm{~b}$ & 1,44 a \\
\hline Limoeiro 'Cravo' & $239,73 \mathrm{a}$ & $31,35 \mathrm{a}$ & $9,40 \quad$ c & $\mathrm{a}$ & $0,50 \mathrm{~b}$ & $19,12 \mathrm{a}$ & $1,22 \mathrm{a}$ \\
\hline CV (\%) & 9,79 & 12,28 & 3,46 & & 5,72 & 5,18 & 12,66 \\
\hline
\end{tabular}

Médias seguidas da mesma letra minúscula na coluna pertencem ao mesmo agrupamento pelo teste de Scott-Knott (5\%).

* Frutos coletados nos meses de abril/maio

** $\mathrm{kg}$ de sólidos solúveis por caixa de $40,8 \mathrm{~kg}$

\section{CONCLUSÕES}

Em pomares irrigados de tangerineira 'Span Americana', os porta-enxertos trifoliata 'Davis A' e HRS 849 apresentam desempenho horticultural satisfatório. Para plantios em alta densidade, a melhor performance da tangerineira 'Span Americana' é obtida com a utilização dos porta-enxertos trifoliata 'Flying Dragon' e citranges 'Troyer' e 'Carrizo'.

\section{AGRADECIMENTOS}

À Fundação de Amparo à Pesquisa do Estado de São Paulo (FAPESP), pelo auxílio financeiro à pesquisa (Processo 2004/16077-3).

\section{REFERÊNCIAS}

ABEDI GHESHLAGHI, E.; FIFAEI, R. Effect of planting density on quantitative and quality traits of Unshiu mandarin (Citrus inshiu) on Flying Dragon rootstock. Seed and Plant Production Journal, Karaj, v. 28, n. 1, p. 81-93, 2012.

AGRIANUAL: anuário da agricultura brasileira, São Paulo: Editora FNP, 2013. p.270-281.

BOWMAN, K.D. Performance of 'Fallglo' citrus hybrid on ten rootstocks in Lake County. Proceedings of the Florida State Horticultural Society, Orlando, v. 111, p. 177-180, 1998.
CANTUARIAS-AVILÉS, T.; MOURÃO FILHO, F.A.A.; STUCHI, E.S.; SILVA, S.R.; ESPINOZANUNEZ, E. Tree performance and fruit yield and quality of 'Okitsu' Satsuma mandarin grafted on 12 rootstocks. Scientia Horticulturae, Amsterdam, v. 123, n. 3, p. 318-322, 2010.

CASER, D. V.; AMARO, A. A. Tangerina: tendências de cultivo no Estado de São Paulo. Análises e Indicadores do Agronegócio, São Paulo, v.1, n.12, 2006.

CAVALCANTE, I.H.L.; MARTINS, A.B.G.; STUCHI, E.S.; CAMPOS, M.C.C. Fruit maturation as a parameter for selection of sweet orange cultivars in Brazil. Journal od Food Agriculture and Environment, Helsinki, v. 7, n. 3-4, p. 316-319, 2009.

FIGUEIREDO, J.O.; NEGRI, J.D.; MATTOS JÚNIOR, D.; PIO, R.M.; AZEVEDO, F.A.; GARCIA, V.X.P. Comportamento de 16 porta-enxertos para o tangor Murcott na região de Itirapina-SP. Revista Brasileira de Fruticultura, Jaboticabal, v. 28, n. 1, p. 76-78, 2006.

GEORGIOU, A. Performance of 'Nova' mandarin on eleven rootstocks in Cyprus. Scientia Horticulturae, Amsterdam, v. 84, n. 1-2, p. 115-126, 2000.

GONZATTO, M.P.; KOVALESKI, A.P.; BRUGNARA, E.C.; WEILER, R.L.; SARTORI, I.A.; LIMA, J.G.; BENDER, R.J.; SCHWARZ, S.F. Performance of 'Oneco' mandarin on six rootstocks in South Brazil. Pesquisa Agropecuária Brasileira, Brasília, v. 46, n. 4, p. 406-411, 2011. 
MATTOS JÚNIOR, D.; DE NEGRI, J.D.; PIO, R.M.; POMPEU JÚNIOR, J. (Org.). Citros. Campinas: Instituto Agronômico/Fundag, 2005. v. 1, 929 p.

MONSELISE, S. P.; GOLDSCHMIDT, E.E. Alternate Bearing in Fruit Trees. Horticultural Reviews, Westport, v. 4, p. 128-173, 1982.

MOURÃO-FILHO, F.A.A.; ESPINOZA-NÚÑEZ, E.; STUCHI, E.S.; ORTEGA, E.M.M. Plant growth, yield, and fruit quality of 'Fallglo' and 'Sunburst' mandarins on four rootstocks. Scientia Horticulturae, Amsterdam, v. 114, n.1, p. 45-49, 2007.

NÚÑEZ, E.E.; MOURÃO FILHO, F.A.A.; STUCHI, E.S. Desenvolvimento vegetativo, produção e qualidade de frutos da tangerina 'Fremont' sobre quatro porta-enxertos. Revista Brasileira de Fruticultura, Jaboticabal, v. 29, n. 2, p. 308-312, 2007.

NÚÑEZ, E.E.; MOURÃO-FILHO, F.A.A.; STUCHI, E.S.; ORTEGA, E.M.M. Desenvolvimento e produtividade da tangerina 'Fairchild' sobre quatro porta-enxertos. Ciência Rural, Santa Maria, v. 38, n. 6, p. 1553-1557, 2008.

PIO, R. M.; KEIGO, M.; FIGUEIREDO, J. O. de. Características do fruto da variedade Span Americana (Citrus reticulata Blanco): uma tangerina do tipo 'Poncã' de maturação precoce. Revista Brasileira de Fruticultura, Jaboticabal, v. 23, n. 2, p. 325-329, 2001.

POMPEU JÚNIOR, J. Porta-enxertos. In: MATTOS JÚNIOR, D. et al. (Ed.). Citros. Campinas: Instituto Agronômico/Fundag, 2005. v. 1, p. 63-104.

POMPEU JÚNIOR, J. Porta-enxertos para citros potencialmente ananicantes. Laranja, Cordeirópolis, v. 22 , n. 1, p. 147-155, 2001.
POMPEU JÚNIOR, J.; LARANJEIRA, F.F.; BLUMER, S. Laranjeiras 'Valência' enxertadas em híbridos de trifoliata. Scientia Agricola, Piracicaba, v. 59, n. 1, p. 93-97, 2002.

SILVA, S.R.; OLIVEIRA, J.C.; STUCHI, E.S.; REIFF, E.T. Qualidade e maturação de tangerinas e seus híbridos em São Paulo. Revista Brasileira de Fruticultura, Jaboticabal, v. 31, n. 4, p. 977-986, 2009.

SMITH, M.W.; SHAW, R.G.; CHAPMAN, J.C.; OWEN-TURNER, J.; LEE, L.S.; McRAE, K.B.; JORGENSEN, K.R.; MUNGOMERY, W.V. Longterm performance of 'Ellendale' mandarin on seven commercial rootstocks in sub-tropical Australia. Scientia Horticulturae, Amsterdam, v. 102, n.1, p. 75-89, 2004.

STENZEL, N.M.C.; NEVES, C.S.V.J.; GOMES, J.C.; MEDINA, C.C. Performance of 'Ponkan' mandarin on seven rootstocks in Southern Brazil. Hortscience, St. Joseph, v. 38, n. 2, p. 176-178, 2003.

STUCHI, E.S.; ESPINOZA-NÚÑEZ, E.; MOURÃO FILHO, F.A.A.; ORTEGA, E.M.M. Vigor, produtividade e qualidade de frutos de quatro tangerineiras e híbridos sobre quatro porta-enxertos. Revista Brasileira de Fruticultura, Jaboticabal, v. 30, n. 3, p. 741-747, 2008.

TAKAHARA, T.; OGATA, T.; FUJISAWA, H.; MURAMATSU, N. Effect of rootstocks on tree growth, yield and fruit quality of 'Shirawaka' Satsuma mandarin (Citrus unshiu Marc.). Bulletin of the Fruit Tree Research Station, Kannon-Dai, v. 35, p. 99-107, 2001.

ZEKRI, M. Citrus rootstocks affect scion nutrition, fruit quality, growth, yield and economical return. Fruits, Paris, v. 55, p. 231-239, 2000. 